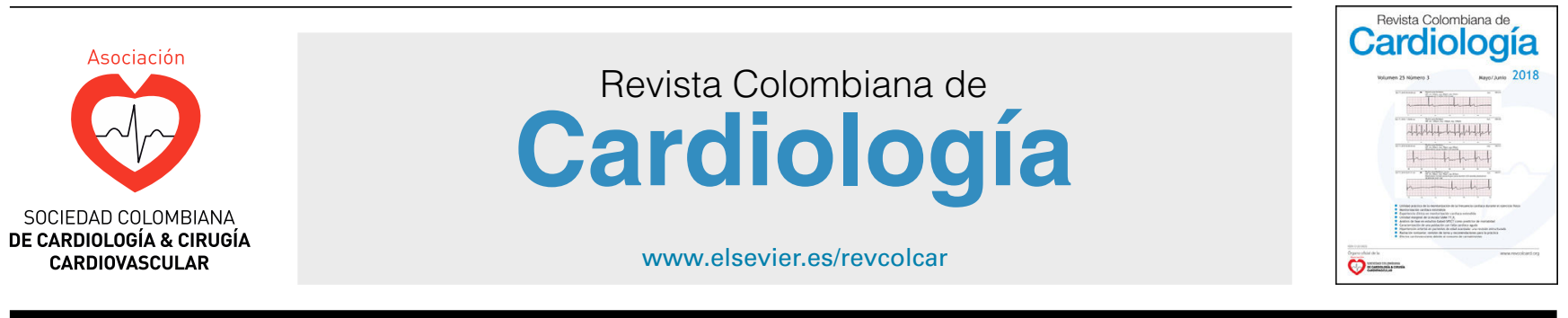

CARDIOLOGÍA DEL ADULTO - REVISIÓN DE TEMAS

\title{
Impacto del uso de edulcorantes no calóricos en la salud cardiometabólica
}

\section{Fernando Manzur-Jattin ${ }^{a}$, María Morales-Núñez ${ }^{a}$, José Ordosgoitia-Morales ${ }^{a}$, Rosalinda Quiroz-Mendoza ${ }^{a}$, Yancarlos Ramos-Villegas ${ }^{\mathrm{a}, \mathrm{b}, *}$ y Hugo Corrales-Santander ${ }^{\mathrm{c}}$}

\footnotetext{
a Centro de Investigaciones Biomédicas (CIB), Facultad de Medicina - Universidad de Cartagena, Cartagena, Colombia

b RED LATINO Organización Latinoamericana de Trauma y cuidado Neurointensivo. Bogotá, Colombia

c Departamento de Medicina, Universidad del Norte. Barranquilla, Colombia
}

Recibido el 29 de julio de 2018; received in revised form 14 de junio de 2019; aceptado el 13 de noviembre de 2019 Disponible en Internet el 17 de abril de 2020

\section{PALABRAS CLAVE}

Edulcorantes no calóricos;

Síndrome metabólico; Salud cardiometabólica
Resumen Los edulcorantes son sustancias capaces de sustituir el dulzor característico del azúcar y en la actualidad son muy utilizados por su capacidad edulcorante, superior a la de la sacarosa, lo que hace que se requieran menos cantidades de estos en los alimentos. Esta característica ha despertado el interés de muchos investigadores por sus posibles beneficios para pacientes con diabetes y obesidad, entre otras morbilidades. En este artículo se hace una revisión acerca de la relación de los edulcorantes no calóricos con el control del peso, el síndrome metabólico y la aparición de eventos cardiovasculares.

(c) 2019 Sociedad Colombiana de Cardiología y Cirugía Cardiovascular. Publicado por Elsevier España, S.L.U. Este es un artículo Open Access bajo la licencia CC BY-NC-ND (http:// creativecommons.org/licenses/by-nc-nd/4.0/).

Impact of the use of calorie-free sweeteners on cardiometabolic health

\section{KEYWORDS}

Calorie-free sweeteners; Metabolic syndrome; Cardiometabolic health

\begin{abstract}
Sweeteners are substances capable of replacing the characteristic sweetness of sugar. They are currently widely used for their sweetener capacity, which is greater than that of saccharose, and which means lower quantities are required in foods. This characteristic has aroused the interest of many researchers due to their possible benefits in diabetes and obesity, as well as in other disorders. A review is presented in this article on the relationship between calorie-free sweeteners and weight control, metabolic syndrome, and the appearance of cardiovascular events.

(c) 2019 Sociedad Colombiana de Cardiología y Cirugía Cardiovascular. Published by Elsevier España, S.L.U. This is an open access article under the CC BY-NC-ND license (http:// creativecommons.org/licenses/by-nc-nd/4.0/).
\end{abstract}

\section{Introducción}

\footnotetext{
* Autor para correspondencia.

Correo electrónico: y-rv@hotmail.com (Y. Ramos-Villegas).
}

Los edulcorantes son sustancias capaces de sustituir el dulzor característico del azúcar. Pueden clasificarse según 
su origen (naturales-artificiales), su contenido calórico (calóricos-no calóricos) o su estructura química (carbohidratos, alcoholes de azúcares, glucósidos) ${ }^{1}$.

A partir de esta clasificación pueden ser tanto naturales como artificiales; entre los naturales acalóricos están la stevia, el luo han guo, la taumatina y la brazzeína ${ }^{1}$, los cuales no tienen índice glucémico, es decir, no alteran la glucosa en sangre porque no son carbohidratos; además, son considerados edulcorantes de alta intensidad, pues su capacidad para endulzar los alimentos es mayor que la de la sacarosa ${ }^{2}$. Por otro lado, los edulcorantes artificiales, como tal, se caracterizan por no hacer un aporte energético, no alterar los niveles de glicemia en sangre y tener gran capacidad edulcorante ${ }^{3}$; en este grupo se encuentran el aspartamo, la sucralosa, la sacarina, I neotamo, el acesulfamo K, el ciclamato, la nehosperidina DC, el alitamo y el advantamo ${ }^{4}$.

\section{Métodos}

Se realizó una búsqueda de información sobre el uso de edulcorantes y su impacto en la salud cardiometabólica, en las bases de datos Science Direct, Scopus y PubMed, a través de los términos MeSH "Nonnutritive Sweetener" and "Obesity" "Metabolic Syndrome" "Cardiovascular Risk"; luego se escogieron los artículos publicados durante los últimos cinco años y a partir de ahí se incluyeron 13 revisiones narrativas, 3 metaanálisis, 3 revisiones sistemáticas, 2 estudios experimentales y 3 estudios de cohorte. Asimismo, se revisaron las referencias de los artículos seleccionados para ampliar la búsqueda.

\section{Propiedades químicas y estructurales de los edulcorantes no calóricos}

Estas varían de acuerdo con la naturaleza de cada edulcorante. La Stevia, por ejemplo, es una planta nativa de Sudamérica, que posee propiedades edulcorantes y no aporta calorías, por lo cual es considerada como medicinal. Las principales sustancias que le otorgan estas propiedades son el esteviósido y el rebaudiósido $A^{2}$. El diterpeno glucósido esteviósido es el que se encuentra en mayor cantidad en las hojas de esta planta y se considera que es 300 veces más dulce que la sacarosa; sin embargo, tiene un regusto amargo, característica que le confiere desventaja. Por otro lado, el rebaudiósido A es la segunda sustancia más abundante en las hojas de la Stevia rebaudiana, y es el más dulce de la familia de los rebaudiósidos, aproximadamente 180 a 400 veces más que la sacarosa ${ }^{5}$.

La taumatina y la brazzeína hacen parte del grupo de las proteínas dulces, las cuales poseen perfiles de gusto lentos $^{2}$. La taumatina se obtiene de la pulpa que recubre las semillas de la planta conocida como "fruto milagroso del Sudán"; esta representa a un grupo de polipéptidos y es considerada como la sustancia más dulce conocida hasta el momento, que brinda un dulzor de larga duración en la boca ${ }^{4}$. Estas sustancias están conformadas por 207 aminoácidos, entre los cuales no se encuentra la histidina; además, tienen gran presencia de puentes disulfuros, los cuales le brindan sostén, estabilidad y termorresistencia.
Su estructura tridimensional es la responsable de su sabor dulce ${ }^{1,4}$.

La sacarina, por su parte, fue el primer edulcorante artificial sintetizado, descubierto casi al azar. Su dulzor es 300 veces mayor que el de la sacarosa, es sensible al calor y tiene un regusto amargo si se utiliza en alta concentración ${ }^{1}$. Su estructura molecular es la de una sulfamida y posee un hidrógeno ligeramente ácido; adicionalmente, esta sustancia es capaz de formar sales con facilidad ${ }^{6}$.

El ciclamato fue descubierto en 1937 y es el menos potente de los edulcorantes artificiales, pues solo es 40 veces más dulce que la sacarosa, razón por la cual suele mezclarse con otros edulcorantes, como la sacarina. Es una sustancia termoestable, de larga vida para su almacenamiento y se caracteriza por ser muy soluble en agua ${ }^{2}$.

La sucralosa es una forma de sacarosa modificada mediante halogenación selectiva; su aporte calórico es de cero y tiene un sabor de hasta 600 veces más dulce que la sacarosa. Es muy soluble en agua y de pobre absorción gastrointestinal ${ }^{4,6}$.

El aspartamo se descubrió en el año 1965; es un metil éter del dipéptido, conformado por los aminoácidos L-aspartato y L-fenilalanina ${ }^{7}$. Aporta 4 calorías por gramo y su sabor es 180 a 200 veces más dulce que la sacarosa ${ }^{8}$.

El acesufalmo de potasio se deriva del ácido acetoacético, y es una sal potásica ligeramente soluble en agua, cuyo dulzor es de entre 160 a 220 veces mayor que el de la sacarosa, y no se metaboliza en el organismo, por lo cual es excretado en la orina sin sufrir cambios ${ }^{1,2,9}$.

Por último, el neotamo es un dipéptido que se deriva del aspartamo; su estructura es similar a este, con la diferencia que se reemplaza el grupo amino del aspartato por un grupo 3,3-dimetilbutilo ${ }^{6}$. Su poder edulcorante es de 8.000 a 13.000 veces más que la sacarosa y es termorresistente, no aporta calorías y su índice glicémico es nulo ${ }^{2}$. A diferencia del aspartamo, es seguro para pacientes con fenilcetonuria, pues su metabolismo no produce fenilalanina ${ }^{1}$.

\section{Fisiopatología de los edulcorantes no calóricos}

El consumo de bebidas azucaradas se ha asociado cada vez más con efectos negativos en el estado de salud, los cuales causan obesidad, diabetes mellitus tipo 2 o síndrome metabólico y, además, constituyen factores de riesgo importantes para el desarrollo de enfermedad cardiovascular ${ }^{10}$. Dada la existencia de esa asociación se propuso en un principio el uso de edulcorantes no calóricos debido a la ventaja de proveer un sabor dulce, sin aportar calorías o efectos glicémicos, y porque eran vistos como una alternativa más saludable para su uso en bebidas y alimentos con el objetivo de disminuir la densidad calórica de los mismos, así como el consumo de energía ${ }^{11}$; sin embargo, recientes estudios han concluido que estos productos no son tan inocuos y saludables como se pensaba y pueden incluso tener efectos poco claros respecto a la regulación del balance energético ${ }^{12}$.

Se han planteado al menos dos hipótesis que podrían explicar esta paradoja entre el consumo de edulcorantes no calóricos y los resultados metabólicos adversos. La primera es la teoría de la causalidad inversa, que consiste en que los individuos con mayor riesgo de ganar peso eligen consumir edulcorantes no calóricos como estrategia para 
reducir el consumo de azúcar y calorías, lo cual constituye un problema a la hora de interpretar los resultados de los diferentes estudios ${ }^{10}$. La segunda consiste en que los edulcorantes no calóricos no son fisiológicamente inertes y afectan los procesos biológicos que intervienen en la regulación de la energía y la homeostasis de la glucosa ${ }^{11}$, evidencia que ha sido avalada por estudios experimentales en modelos animales y humanos que han dilucidado múltiples mecanismos biológicos que podrían explicar dicha asociación ${ }^{13}$.

Entre los diferentes mecanismos fisiopatológicos a través de los cuales estos podrían desencadenar las distintas alteraciones metabólicas se ha propuesto que podrían interferir con las respuestas fisiológicas aprendidas que contribuyen a controlar la glucosa y la homeostasis energética, alterar la microbiota intestinal e inducir intolerancia a la glucosa ${ }^{11}$ e interactuar con receptores del gusto en el sistema digestivo que desempeñan un papel en la absorción de glucosa y la secreción de insulina ${ }^{12,14}$ (fig. 1).

\section{Los edulcorantes no calóricos y el riesgo de eventos cardiovasculares}

Existen estudios prospectivos no experimentales que han documentado asociaciones entre el consumo de bebidas endulzadas con edulcorantes no calóricos y enfermedades crónicas, como la enfermedad arterial coronaria, la insuficiencia renal crónica y el síndrome metabólico. Sin embargo, para estas cohortes de estudios, organizaciones internacionales como la American Heart Asssociation (AHA, su sigla en inglés) y la American Diabetes Association (ADA, su sigla en inglés) consideran posible que el origen de dichas relaciones sea secundario al fenómeno de la causalidad inversa ${ }^{15}$. En contraposición, existen trabajos, como el CARDIA study en el que se concluyó que aquellas personas consumidoras de bebidas con edulcorantes no calóricos tienen menos posibilidades de presentar niveles de glucosa o LDL elevados ${ }^{16}$, o el Woman Health Iniciative en el cual se evaluó el consumo de bebidas dietéticas y el riesgo de eventos cardiovasculares y concluyó que aquellas mujeres posmenopáusicas que consumieron dos o más bebidas dietéticas diariamente tuvieron un aumento significativo de eventos cardiovasculares (HR:1,3; IC:1,1-1,5) y de mortalidad por enfermedad cardiovascular (HR 1,5;IC:1,03-2,3) $)^{17}$.

Por último, entre los estudios más actuales cabe mencionar el de Azad et al. en el cual se realizó una revisión sistemática y metaanálisis de ensayos clínicos aleatorizados y estudios de cohorte en el que la alta ingesta de edulcorantes no calóricos se relacionó con riesgos más altos de hipertensión arterial (cociente de riesgo HR: 1,13; IC 95\%: 1,06-1,20), accidente cerebrovascular (RR: 1,14; IC 95\%: 1,04 - 1,26), eventos cardiovasculares (RR: 1,32; IC 95\%: $1,15-1,52$ ) y nuevos casos de diabetes de tipo 2 (RR: 1, 14; IC 95\%: $1,05-1,23)^{18}$. Pese a los resultados descritos anteriormente, la evidencia actual es insuficiente para establecer si existe o no asociación entre los edulcorantes no calóricos dada la poca cantidad de ensayos controlados aleatorizados en humanos que exploren el papel de estos en el mantenimiento de un peso saludable y la minimización del riesgo metabólico.

\section{Impacto del consumo de edulcorantes no calóricos} en la glicemia

En la actualidad el uso de los edulcorantes no calóricos ha aumentado en el mundo. Antes se creía que los edulcorantes no calóricos no tenían funciones metabólicas, pero la evidencia ha demostrado lo contrario, pues se ha hallado una relación entre el consumo de estos y la intolerancia a la glucosa ${ }^{14}$. Un metaanálisis realizado por Immamura et al. en pacientes con diabetes tipo 2 , mostró un $25 \%$ de aumento en el riesgo de sufrir diabetes asociado al consumo de una porción de edulcorantes no calóricos al día ${ }^{14}$, mientras que Nichol et al. no encontraron aumento de los niveles de glicemia luego del consumo de edulcorantes no calóricos, y, por el contrario, sí evidenciaron una disminución gradual de estos niveles ${ }^{19}$.

Se han planteado diversos mecanismos por los cuales los edulcorantes no calóricos producen intolerancia a la glucosa. Entre estos se destacan las alteraciones de la microbiota intestinal y la interferencia de receptores, como T1R2 Y T1R3, que se encuentran a lo largo del tracto intestinal; estos producirían liberación de insulina, neurotransmisores y otras hormonas ${ }^{14,20}$.

Los edulcorantes no calóricos tienen la capacidad de estimular los receptores T1R2 y T1R3 de las células de revestimiento del tracto intestinal ${ }^{14}$; estos se encuentran presentes en las células L secretoras de GLP1 y producen aumento en la liberación de la insulina e impacto glucémico ${ }^{10}$. Estudios recientes han demostrado el impacto del uso de edulcorantes, disminuyendo la liberación de GLP1 durante las pruebas de tolerancia oral a la glucosa e hiperinsulinemia ${ }^{21}$; Mace et al. demostraron que la presencia de $1 \mathrm{mmol} / \mathrm{L}$ de sucralosa o acesufalme $\mathrm{K}$ aumentó el transporte de glucosa a través de GLUT2, es decir, que el uso de los edulcorantes interviene en el aumento del transporte y absorción de la glucosa ${ }^{14}$. Entre tanto, un estudio realizado por Grotz et al. no mostró ningún efecto de la ingesta de sucralosa en la homeostasis de glucosa $^{19}$.

Adicionalmente, otro de los mecanismos implicados es la secreción de incretinas. En este sentido, un estudio realizado por Jang et al. evaluó la respuesta secretora de GLP-1 frente al uso de acesufalme $K$, sucralosa y sacarina, y en todos los casos observó aumento en la secreción de incretinas; sin embargo, el edulcorante con la capacidad secretora máxima fue el acesufalme $\mathrm{K}^{14}$.

Generalmente, el mecanismo por el cual interfieren en la homeostasis de la glucosa es similar entre los edulcorantes no calóricos, por ende, intervienen otros aspectos, como edad, comorbilidades (principalmente diabetes) y peso corporal ${ }^{22}$, y si bien existen muchos estudios, aún no se han determinado los mecanismos específicos y el efecto que producen los edulcorantes en el control glicémico.

\section{Relación entre edulcorantes no calóricos y control del peso}

Las causas de sobrepeso y obesidad son multifactoriales; no obstante, con su rápido incremento de la prevalencia la evidencia científica ha implicado una serie de factores 


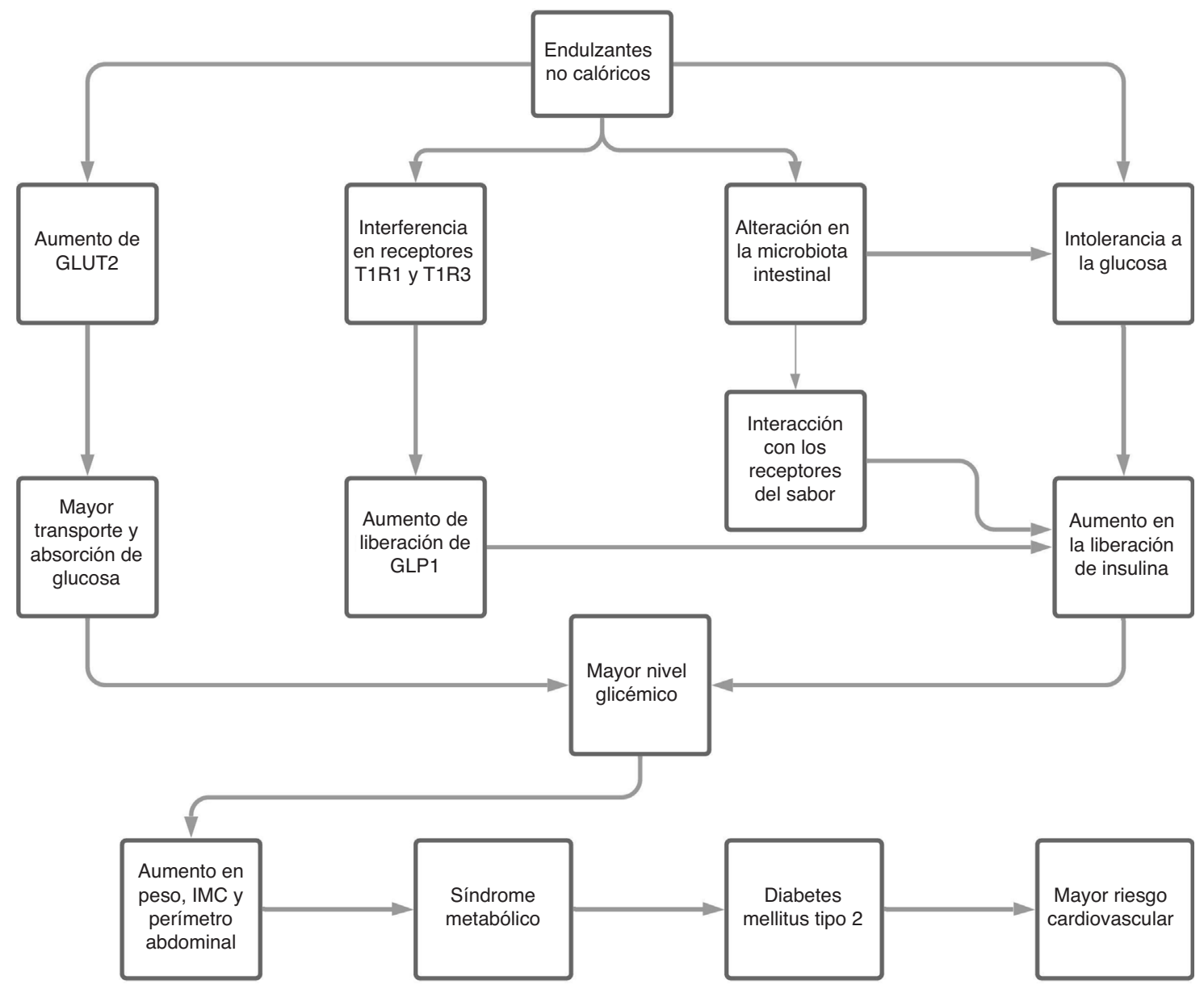

Figura 1 Alteraciones fisiopatológicas de los edulcorantes no calóricos y su relación con el aumento del riesgo cardiovascular. Diseñado por: Ramos-Villegas Y.

dietéticos como posibles contribuyentes; entre estos se encuentra el alto consumo de azúcares ${ }^{13}$. Dado que reducir el consumo de edulcorantes en las comidas y bebidas ha demostrado no ser un objetivo fácil de alcanzar, se han implementado estrategias dietéticas en las que se incluyen los edulcorantes no calóricos como una alternativa "saludable" que promete una disminución del sobrepeso y la obesidad, hecho que actualmente carece de una evidencia científica clara y consecuente ${ }^{13}$. Entre los diferentes estudios que respaldan lo anterior está el de Miller et al. en el que se concluye que el uso de edulcorantes no calóricos pueden ser beneficioso a corto plazo para disminuir el peso corporal $(-0,80 \mathrm{~kg}$; $95 \% \mathrm{Cl}:-1,17$, $-0,43$ ), el índice de masa corporal [BMl (en $\mathrm{kg} / \mathrm{m}^{2}$ ): $0,24 ; 95 \% \mathrm{Cl}:-0,41,-0,07]$ y la circunferencia abdominal $(-0,83 \mathrm{~cm} \text {; } 95 \% \mathrm{Cl}:-1,29,-0,37)^{3}$; sin embargo, en contraposición se encuentra el estudio de Azad et al. en el cual la alta ingesta de edulcorantes no calóricos se relacionó en forma estadísticamente significativa con aumentos moderados a largo plazo del peso corporal, el índice de masa corporal y el perímetro de la cintura ${ }^{18}$. Así, pues, la evidencia actual no es concluyente y resulta ser contradictoria.

\section{Relación entre síndrome metabólico y consumo de edulcorantes no calóricos}

El uso de bebidas azucaradas ha sido asociado de manera creciente con el sobrepeso, la obesidad, la diabetes mellitus tipo 2 y el síndrome metabólico; por consiguiente, recientemente se ha hecho más común el uso de endulzantes no calóricos $^{23}$. Antes de la aprobación de su uso por la FDA múltiples estudios toxicológicos se hicieron con base en estas sustancias, y concluyeron que son seguras y bien toleradas; además, se encontró que, aún a grandes dosis, el consumo de sucralosa y aspartame no se asoció con alteraciones en valores de glucosa, hemoglobina glicosilada y péptido $C$, tanto en pacientes diabéticos como no diabéticos ${ }^{11}$. Si bien, en teoría, la ingesta menor de calorías podría representar un beneficio, también se deben tener en cuenta los efectos contrarios asociados al menor consumo de energía y menor ganancia de peso; así mismo, sus efectos benéficos sobre el síndrome metabólico aún no están claros $^{12}$. Algunos estudios han demostrado que el consumo de edulcorantes no calóricos no se ha asociado con mejores condiciones clínicas; incluso, en algunos casos se han asociado con aumento de peso, síndrome metabólico y diabetes tipo $2^{11}$. 
Tabla 1 Comparación IDA según FDA y EFSA

\begin{tabular}{ll}
\hline Edulcorantes no calóricos & \\
\hline Aspartamo & 5040 \\
Sucralosa & 515 \\
Sacarina & 155 \\
Neotamo & 0,32 \\
Acesufalme K & 159 \\
Ciclamato & No aprobado 7 \\
Nehosperidina c & No aprobado 5 \\
Advantamo & 32,85
\end{tabular}

Adaptado de Serra-Majem et al. ${ }^{25}$

Pepino describió tres posibles mecanismos que explican los efectos metabólicos de los edulcorantes no calóricos:

1) Alteraciones en la respuesta neuroendocrina ante el consumo de alimentos con sabor dulce, lo cual afecta el metabolismo de la glucosa y de las grasas, y predispone a ganancia de tejido adiposo.

2) Afectación de la microbiota intestinal, que causa niveles más altos de glicemia al momento de la ingesta de glucosa, y por ende, desarrollo de intolerancia a esta.

3) Interacción con los receptores de sabor dulce en el intestino, que altera la respuesta de la insulina ante la ingesta de glucosa ${ }^{11}$.

Por otro lado, existen algunos estudios que sugieren el efecto negativo que podrían tener los edulcorantes no calóricos en la salud de sus consumidores. Hess et al. encontraron que el consumo de edulcorantes no calóricos en general y aspartame solo es predictor significativo para la aparición de síndrome metabólico ${ }^{24}$.

Karaletzi et al. realizaron una revisión sistemática con un total de 13 estudios acerca de la relación entre el consumo de edulcorantes y un impacto negativo en el metabolismo, cuyos resultados mostraron un $15 \%$ de riesgo de aumento en el índice de masa corporal con el consumo de edulcorantes; sin embargo, la evaluación de la literatura no evidenció una asociación entre el consumo de edulcorantes no calóricos y otros componentes del síndrome metabólico, como circunferencia de la cintura, acumulación de tejido adiposo y diabetes mellitus tipo $2^{6}$. Figlewicz et al., por su parte, realizaron un estudio en modelos animales y encontraron que la ingesta de edulcorantes no calóricos, aun en moderada cantidad, se asocia de forma similar a la ingesta de bebidas azucaradas pero con alteración en los patrones alimenticios, hecho que predispone al aumento de peso; además, encontraron discretas alteraciones en los niveles de lípidos en sangre ${ }^{23}$.

\section{Recomendaciones acerca del uso de edulcorantes no calóricos}

Según la Asociación Latinoamericana de Diabetes (ALAD) el consumo de edulcorantes no calóricos se hace con base en los niveles de ingesta diaria aceptados (IDA), establecidos por la FDA (Administración de Alimentos y Medicamentos) y la EFSA (Autoridad Europea de Seguridad alimentaria) 25,26 (tabla 1).
Por otro lado, un consenso iberoamericano realizado en 2018 creó las siguientes pautas en cuanto al consumo de edulcorantes no calóricos ${ }^{25,26}$ :

- El uso de edulcorantes no calóricos en pacientes con obesidad contribuye a disminuir y mantener del peso perdido siempre y cuando esté asociado a un plan dietético estructurado.

- El uso de edulcorantes no calóricos en programas de control de diabetes puede contribuir a un mejor control glicémico.

- Los edulcorantes no calóricos son aptos para el consumo de la población general, y se recomiendan en pacientes diabéticos, con sobrepeso, historia de caries dental severa o cualquier condición que restringa el uso de azúcares.

- El consumo de edulcorantes no calóricos es apto en pacientes embarazadas y mujeres lactantes, siempre que las ingestas no excedan los IDA.

\section{Conclusión}

En el pasado se pensaba que el uso de edulcorantes no calóricos aportaba beneficios en la salud de los consumidores debido a su inactividad metabólica. Sin embargo, desde hace muchos años este concepto ha cambiado y en muchos estudios se ha evidenciado una asociación entre el uso de edulcorantes no calóricos y alteraciones metabólicas, como intolerancia a la glucosa, eventos cardiovasculares y síndrome metabólico. A lo largo de esta revisión se observa que los estudios presentan resultados contradictorios, por lo cual se hace necesario emprender estudios con mejor nivel de evidencia en el campo de los edulcorantes no calóricos y su relación con la salud cardiometabólica. Mientras tanto deben tenerse en cuenta las recomendaciones para el uso de edulcorantes no calóricos emitidas por la ALAD, la ADA y el Consenso iberoamericano sobre edulcorantes bajos en calorías y sin calorías: seguridad, aspectos nutricionales y beneficios en alimentos y bebidas.

\section{Financiación}

El presente artículo se realizó gracias al apoyo de la vicerrectoria de investigaciones de la Universidad de Cartagena a los grupos de Investigación de la Facultad de Medicina a través de los planes de fortalecimiento de grupos.

\section{Conflicto de intereses}

Ninguno.

\section{Bibliografía}

1. García-Almeida JM, Fdez GMC, Alemán JG. Una visión global y actual de los edulcorantes Aspectos de regulación. Nutr Hosp. 2013;28 suppl.4:17-31.

2. Mooradian AD, Smith $M$, Tokuda $M$. The role of artificial and natural sweeteners in reducing the consumption of table sugar: A narrative review. Clin Nutr ESPEN. 2017;18:1-8. 
3. Miller PE, Perez V. Low-calorie sweeteners and body weight and composition: a meta- analysis of randomized controlled trials and prospective cohort studies. Am J Clin Nutr. 2014:1-13.

4. Raúl CL, Nelly AB. Características de los edulcorantes no calóricos y su uso en niños. Acta Pediátrica México. 2014;34:141-53.

5. Hurtado-Ibarbo JH, Arroyave-Roa JD, Cardona-Caicedo M. Endulzantes naturales utilizados en la elaboración de chocolate. 2017;15:142-52.

6. Non-Nutritive Sweeteners and Metabolic Health Outcomes in Children. J Pediatr. 2018;197:128-133.e2.

7. Marinovich M, Galli CL, Bosetti C, Gallus S, La Vecchia C. Aspartame, low-calorie sweeteners and disease: Regulatory safety and epidemiological issues. Food Chem Toxicol. 2013;60:109-15.

8. Chil R, Vol N, Apetito, ganancia de peso Non-nutritive sweeteners risks. appetite and weight gain. 2013;40(5.).

9. Bian X, Chi L, Gao B, Tu P, Ru H, Lu K. The artificial sweetener acesulfame potassum affects the gut microbiome and body weight gain in CD-1 mice. PLoS One. 2017;12:1-9.

10. Pereira MA, Odegaard AO. Artificially sweetened beverages do they influence cardiometabolic risk? Current Athosclerosis Report. 2013;13:375.

11. Pepino MY. Physiology and behavior metabolic effects of nonnutritive sweeteners. Physiol Behav. 2015;152:450-5.

12. Swithers SE. Artificial sweeteners produce the counterintuitive effect of inducing metabolic derangements. Trends Endocrinol Metab. 2013;24:431-41.

13. Swithers SE. Artificial sweeteners are not the answer to childhood obesity. Appetite. 2015;93:85-90.

14. Chan CB, Hashemi Z, Subhan FB. The impact of low and nocaloric sweeteners on glucose absorption, incretin secretion, and glucose tolerance. 2017;9:1-9.

15. Aldrete Velasco J, López-García R, Zúñiga-Guajardo S, RiobóServán P, Serra-Majem L, Suverza-Fernández A. Análisis de la evidencia disponible para el consumo de edulcorantes no calóricos Documento de expertos. Med Int Mex. 2017;33:61-83.

16. Duffey KJ, Steffen LM, Horn L, Van, Jacobs DR, Popkin BM. Dietary patterns matter: diet beverages and cardiometabolic risks in the longitudinal Coronary Artery Risk Development in Young Adults (CARDIA) Study 1-3. Am J Clin Nutr. 2012;95:909-15.

17. Vyas A, Rubenstein L, Robinson J, Seguin RA, Vitolins MZ, Kazlauskaite $\mathrm{R}$, et al. Diet drink consumption and the risk of cardiovascular events: A Report from the Women's Health Initiative. J Gen Intern Med. 2015;30:462-8.

18. Azad MB, Abou-Setta AM, Chauhan BF, Rabbani R, Lys J, Copstein L, et al. Nonnutritive sweeteners and cardiometabolic health: a systematic review and meta-analysis of randomized controlled trials and prospective cohort studies. Can Med Assoc J. 2017; 189:E929-39.

19. Russell WR, Baka A, Björck I, Delzenne N, Gao D, Griffiths HR, et al. Impact of diet composition on blood glucose regulation. Crit Rev Food Sci Nutr. 2016;56:541-90.

20. Lertrit A, Srimachai S, Saetung S, Chanprasertyothin S, Chailurkit L, Areevut C, et al. Effects of sucralose on insulin and GLP-1 secretion in healthy subjects: a randomized double-blind, placebo controlled trial. Nutrition. 2018;55-56:125-30.

21. Fowler SPG. Physiology and behavior low-calorie sweetener use and energy balance: results from experimental studies in animals, and large-scale prospective studies in humans. Physiol Behav. 2016;164:517-23.

22. Nichol AD, Holle MJ, Nichol AD. Glycemic impact of nonnutritive sweeteners: a systematic review and meta-analysis of randomized controlled trials. Eur J Clin Nutr. 2018;72:796-804.

23. Figlewicz DP, Ioannou G, Bennett Jay J, Kittleson S, Savard C, Roth CL. Effect of moderate intake of sweeteners on metabolic health in the rat. Physiol Behav. 2009;98:618-24.

24. Hess EL, Myers EA, Swithers SE, Hedrick VE, Hess EL, Myers EA, et al. Associations between nonnutritive sweetener intake and metabolic syndrome in adults. J Am Coll Nutr. 2018;0:1-7.

25. Serra-Majem L, Raposo A, Aranceta-Bartrina J, Varela-Moreiras $\mathrm{G}$, Logue C, Laviada $\mathrm{H}$, et al. Iberoamerican consensus on low-and no-calorie sweeteners: safety, nutritional aspects and benefits in food and beverages. Nutrients. 2018;10:818.

26. Sánchez-Thorin JC, Chica LG, González AL, Bohórquez MC, Lizcano $\mathrm{F}$, Ocampo HH, et al. Panel de expertos sobre la atención inicial de la retinopatía diabética. Rev ALAD. 2018;8:175-83. 